Arch. hist. jap. Vol. 16, n. 4 (April 1959).

S. $599-611$.

Anat. Inst. d. Med. Fak., Univ. Okayama (Vorstand: Prof. M. SEKI).

\title{
Über die Menge von Lipoiden und Mukopolysacchariden in der Arterienwand von Knochen- und Knorpelfisch.
}

\author{
硬骨魚之軟骨魚の動脉壁の類脂質之粘液多糖類の量.
}

Rei HIROSE 広 瀬令.

(Eingegangen am. 31 Januar 1959.)

In der sklerosierten Arterienwand des Menschen vermehren sich bekanntlich Lipoide. Nach der Untersuchung von TOKUDA (1957) nehmen Lipoide auch im Blutplasma der Alten mit erhöhtem Blutdruck zu. Andererseits hat neulich die Beziehung zwischen der Arteriosklerose und den sauren Polysaccharidverbindungen von manchen Forschern Beachtung gefunden. Es wurde von KURITA (1955) konstatiert, daßdie dem Tier einverleibte Chondroitinschwefelsäure, wie ihr verwandtes Heparin, die Cholesterinmenge im Blut herabsetzt und auf die Atherombildung eindämmend wirkt. KINUKAWA (1957) beobachtete, daß in der Media der Arterien und in der Grundsubstanz des Knorpels des langfristig mit Natriumsulfidlösung intravenös injizierten Kaninchens sich die mit Toluidinblau metachromatisch färbbaren sauren Polysaccharidverbindungen beträchtlich vermehren, dagegen in der Grundsubstanz in der Arterienmedia die mit Irisolechtviolett BBN färbbaren Lipoide auffallend vermindern. Auch wurde der Zusammenhang zwischen der Menge der Lipoide und der sauren Polysaccharide im Tierkörper bei verschiedenen normalen Tieren untersucht. Vor allem wurde von TOKUDA (1957) bemerkt, daß die Menge der Lipoide im Blutplasma bei den Knochenfischen beträchtlich größer ist, demgegenüber die sauren Polysaccharide im Knorpel sich bei den Knorpelfischen bei weitem reichlicher vorhanden sind. Es wurden in der vorliegenden Arbeit die Menge der Lipoide und der Mukopolysaccharide in der Arterienwand bei den Knochen- und Knorpelfischen vergleichend untersucht.

\section{Material und Methode.}

Als Versuchsmaterial wählte man folgende 14 Arten von Knochenfischen und 3 Arten von Knorpelfischen. Knochenfisch : Sparus swinhonis GÜNTHER (クロダ イ), Trachurus japonicus TEMMINCK et SCHLEGEL (マアヂ), Scomber japonicus HOUTTUYN (ホンサバ), Cololabis saira BREVOORT (サンマ), Limanda yokohamae GÜNTHER (マガレイ), Lateolabrax japonicus CUVIER et VALENCIENNES (スズキ), Sawara niphonia CUVIER et VALENCIENNES (サワラ), Mugil cephalus LINNÉ(マボラ), Platycephalus indicus LINNÉ(コチ), Paralichthys olivaceus TEMMINCK et SCHLEGEL (ヒラメ), Pagrosomus major TEMMINCK et SCHLEGEL (マダイ), Anguilla japonica TEMMINCK et SCHLEGEL (ウナギ), Seriora quinqueradiata TEMMINCK et SCHLEGEL (ハマチ), Astroconger myriaster BREVOORT (マアナゴ) und Knorpelfisch: Dasyatis akajei MÜLLER et HENLE (アカエイ), 
Rhinobatus Schlegeli MÜLLER et HENLE (サカタザメ), Heterodontus japonicus DUMÉRIL (ネコザメ).

Man entnahm den Fischen die Aorta ventralis und fixierte in 10\%igem Formalin, um $25 \mu$ dicke Gefrierquerschnitte zu verfertigen. Wie in den Tafelabbildungen zu sehen ist, klebte man die zu vergleichenden Schnitte von Knochen- und Knorpelfisch auf ein und demselben Objektglas, so daß man sie unter gleichen Bedingungen färben und weiter behandeln konnte. Zur Darstellung von Lipoiden wurden die Schnitte mit schwach saurem Irisolechtviolett BBN und schwach basischem Viktoriablau gefärbt, und auf Polysaccharidverbindungen wurden die Perjodsäure-SCHIFF-Reaktion (fortan Pjs-SCHIFF-Reaktion) und die metachromatische Färbung mit 0.2\%iger Toluidinblaulösung bei pH 2.5 und pH 4.1 ausgeführt.

\section{Ergebnisse.}

Eingangs sei erwähnt, daß die Wand der Aorta ventralis der Knochenfische, worauf z. B. KRAUSE (1923) bei Esox lucius hingewiesen hat, deutlich in drei Schichten Intima, Media und Adventitia eingeteilt ist. Ihre Intima ist ein dünnes Plattenepithel. Die Media besteht aus mehrlagernden zirkulären Muskeln, zwischen denen wenige feine elastische und kollagene Fasern eingeschaltet sind. In der Adventitia laufen ziemlich dicke elastische Lamellen zirkulär und leicht schlängelnd, welche feinere elastische Fasern abzweigen. Nur bei Sparus swinhonis (クロダイ), Pagrosomus major (マダイ), Mugil cephalus (マボラ) sind die elastischen Fasern in der Media der Arterie netzförmig, wie von KITADE (1957) beobachtet wurde.

In der Wand der Aorta ventralis der Knorpelfische ist auch Intima, Media und Adventitia zu sehen. Die Intima ist sehr dünn. Die Media besteht bei Dasyatis akajei (アカエイ) und Rhinobatus Schlegeli (サカタザメ) wie bei den Knochenfischen aus ringförmig laufenden, ziemlich dicken Muskeln mit geringem Bindegewebe. Die elastischen Fasern finden sich auch gew öhnlich weniger als bei den Knochenfischen. Die elastischen Fasern in der Adventitia verzweigen sich beträchtlich und bilden ein viel komplizierteres und dichteres Netzwerk als beim Knochenfisch. Die Muskeln in der Media sind bei Heterodontus japonicus (ネコザメ), eine Art von Plagiostomen, im Gegensatz zu obigen zwei Bastoiden sehr dünn. Die Adventitia ist aber dick. Ihr kompliziertes dichtes Netzwerk aus elastischen Fasern wird nach innen allmählich locker und geht ohne deutliche Grenze zu der Media über.

\section{A. Die Lipoide.}

1. Scomber japonicus (ホンサバ) (Knochenfisch) und Dasyatis akajei (アカエ 1) (Knorpelfisch).

Vor allem ist zu bemerken, daß sich die elastischen Fasern in der Adventitia der Aorta ventralis dieser beiden Fische mit den lipoidfärbenden Farbstoffen gut färben. Die Menge der so nachzuweisenden Lipoide in der Media ist beim Knochenfisch beträchtlich größer als beim Knorpelfisch. Auch in der amorphen Grundsubstanz in der Adventitia lassen sich die Lipoide beim Knochenfisch etwas mehr nachweisen. Die Färbbarkeit der elastischen Fasern in der Adventitia mit Irisol- 
echtviolett BBN ist fast die gleiche bei beiden Fischen (Abb. 1), aber die mit Viktoriablau bei dem Knochenfisch etwas stärker (Abb. 7).

2. Trachurus japonicus (マアヂ) (Knochenfisch) und Dasyatis akajei (アカエ 1) (Knorpelfisch).

Die amorphe Grundsubstanz in der Media und Adventitia der Aorta des Knochenfisches färbt sich mit Irisolechtviolett BBN und Viktoriablau ziemlich gut, aber dabei die in der Media etwas stärker als in der Adventitia (Abb. 2 und 8).

Dagegen werden die Media und Adventitia bei dem Knorpelfisch mit den lipoidfärbenden Farbstoffen viel schlechter gefärbt:

3. Sparus swinhonis (クロダイ) (Knochenfisch) und Heterodontus japonicus (ネコザメ) (Knorpelfisch).

In der Aortenmedia dieses Knochenfisches sind die mit den lipoidfärbenden Farbstoffen nachweisbaren Lipoide ziemlich reichlich vorhanden (Abb. 9), auch in der amorphen Grnndsubstanz der Adventitia (Abb. 3).

Dagegen sind sie beim Knorpelfisch viel weniger zu finden. Die elastischen Fasern in der Adventitia werden jedoch mit den lipoidfärbenden Farbstoffen ziemlich gut gefärbt.

4. Mugil cephalus (マボラ) (Knochenfisch) und Heterodontus japonicus (ネコ ザメ) (Knorpelfisch).

Die amorphe Grundsubstanz in der Media und Adventitia wird beim Knochenfisch mit den lipoidfärbenden Farbstoffen ziemlich stark gefärbt (Abb. 4). Die Menge der Lipoide ist bei ihm größer als beim Knochnfisch.

5. Cololabis saira (サンマ) (Knochenfisch) und Rhinobatus Schlegeli (サカタ ザメ) (Knorpelfisch).

Die Lipoidemenge in der Media ist beim Knochenfisch auffallend größer als beim Knorpelfisch. Die amorphe Grundsubstanz in der Adventitia färbt sich aber bei beiden Fischen in fast gleicher Stärke (Abb. 5).

6. Lateolabrax japonicus (スズキ) (Knochenfisch) und Heterodontus japonicus (ネコザメ) (Knorpelfisch).

Bei dem Knochenfisch finden sich Lipoide in der Media in viel größerer Menge als beim Knorpelfisch (Abb. 6). In der amorphen Grundsubstanz der Adventitia kommen aber Lipoide bei beiden Fischen beinahe in gleicher Menge vor.

7. Anguilla japonica (ウナギ) (Knochenfisch) und Rhinobatus Schlegeli (サカ タザメ) (Knorpelfisch).

Es werden Lipoide in der Media des Knochenfisches weitaus in größerer Menge nachgewiesen als in der des Knorpelfisches (Abb. 10). Die elastischen Fasern in der Adventitia sind bei diesem Knochenfisch weniger zahlreich als bei dem Knochenfisch. Die Färbbarkeit der amorphen Grundsubstanz der Adventitia mit Irisolechtviolett BBN ist fast die gleiche wie beim Knorpelfisch.

Im folgenden soll die Lipoidenmenge in der Aorten wand an einigen Paaren von Knochenfischen besprochen werden.

In der Aortenmedia von Pagrosomus major (マダイ) (Knochenfisch) und Astroconger myriaster (マアナゴ) (Knochenfisch) befinden sich Lipoide sehr reichlich, aber auch in der amorphen Grundsubstanz der Adventitia sind sie ziemlich reichlich. Die ganze Lipoidmenge in der Arterienwand dieser Knochenfische erweist sich 
als beträchtlich größer als z. B. bei dem Knorpelfisch, Heterodontus japonicus (衤 コザメ).

Bei Sawara niphonia (サワラ) (Knochenfisch) und Seriora quinqueradiata (ハマ チ) (Knochenfisch) findet sich Lipoide in der Media reichlicher als in der Adventitia. Die ganze Lipoidemenge ist viel größer als beim Knorpelfisch, Dasyatis akajei (アカエイ).

Bei Platycephalus indicus (コチ)(Knochenfisch), Limanda yokohamae (マガレ イ) (Knochenfisch) und Paralichthys olivaceus (ヒラメ) (Knochenfisch) wird nur die innere Schicht der Media mit den lipoidfärbenden Farbstoffen schwach gefärbt. Die Lipoidemenge ist also nur um ein weniges größar als beim Knorpelfisch, Dasyatis akajei (アカエイ).

\section{B. Die Pjs-SCHIFF-Reaktion.}

1. Scomber japonicus (ホンサバ) (Knochenfisch) und Dasyatis akajei (アカエ 1) (Knorpelfisch).

Die Pjs-SCHIFF-positive Substanz ist sowohl in der Media als auch Adventitia der Arterienwand beim Knochenfisch beträchtlich reichlicher vorhanden als beim Knorpelfisch (Abb. 11). Die Pjs-SCHIFF-Reaktion tritt immer in der Adventitia stärker auf als in der Media. Bei beiden Fischen erscheinen die elastischen Fasern in der Arterienwand stark rot.

2. Trachurus japonicus (マアヂ) (Knochenfisch) und Dasyatis akajei (アカエ 1) (Knorpelfisch).

Die Pjs-SCHIFF-Reaktion in der Arterienwand tritt beim Knochenfisch viel stärker ein als beim Knorpelfisch (Abb. 12). Bei beiden Fischen erscheint die Reaktion in der Adventitia stärker als in der Media.

3. Sparus swinhonis (クロダイ) (Knochenfisch) und Dasyatis akajei (アカエ १) (Knorpelfisch).

Die Pjs-SCHIFF-positive Substanz wird in der Arterienwand des Knochenfisches reichlicher gefunden als in der des Knorpelfisches (Abb. 13). Bei dem Knochenfisch ist die Stärke und Ausdehnung der Reaktion der amorphen Grundsubstanz in der Media und Adventitia fast gleich.

4. Cololabis saira (サンマ) (Knochenfisch) und Dasyatis akajei (アカエイ) (Knorpelfisch).

Die Pjs-SCHIFF-Reaktion der Media und Adventitia tritt beim Knochenfisch beträchtlich stärker auf als beim Knorpelfisch (Abb. 14).

5. Platycephalus indicus (コチ) (Knochenfisch) und Rhinobatus Schlegeli (サ カタザメ) (Knorpelfisch).

Wie in Abb. 15 zu sehen ist, fällt die Pjs-SCHIFF-Reaktion der Media beim Knochenfisch stärker aus als beim Knorpelfisch, aber die Stärke und Ausdehnung der Reaktion der Adventitia ist bei beiden Fischen fast gleich.

6. Pagrosomus major (マダイ)(Knochenfisch) und Rhinobatus Schlegeli (サカ タザメ) (Knorpelfisch).

Auch bei diesen Fischen tritt die Pjs-SCHIFF-Reaktion der Media wie auch der Adventitia beim Knochenfisch etwas stärker aus als beim Knorpelfisch (Abb. 
16).

Bei den Knochenfischen, Lateolabrax japonicus (スズキ), Mugil cephalus (マボ ラ), Anguilla japonica (ウナギ) und Astroconger myriaster (マアナゴ) ist die PjsSCHIFF-positive Substanz in der Aortenwand mehr vorhanden als z. B. beim Knorpelfisch, Dasyatis akajei (アカエイ). Sie findet sich in der Adventitia mehr als in der Media.

Bei den drei Fischarten, Limanda yokohamae (マガレイ), Sawara niphonia (サ ワラ), Paralichtys olivaceus (ヒラメ), welche alle Knochenfisch sind, ist jedoch die Menge der Pjs-SCHIFF-positiven Substanz in der Media wie auch in der Adventitia fast die gleiche wie bei dem Knorpelfisch, Heterodontus japonicus (ネコザメ).

\section{Die Metachromasie.}

Bei den allen untersuchten Fischen (14 Arten Knochenfisch und 3 Arten Knorpelfisch) tritt die Metachromasie bei der Färbung mit Toluidinblau bei $\mathrm{pH} 4.1$ ein, welche bei den Knochen- und Knorpelfischen immer am stärksten an der Grenze zwischen der Media und Adventitia zutage tritt. Beim Knochenfisch erscheint die Metachromasie bei pH 4.1 in der Media ziemlich stark, in der amorphen Grundsubstanz in der Adventitia aber scwächer. Beim Knorpelfisch tritt dagegen die Metachromasie in der Adventitia beträchtlich stärker auf als in der Media. Von Wichtigkeit ist, daß die metachromatische Färbung der Arterienwand mit Toluidinblau bei pH 4.1 bei den Knorpelfischen, Dasyatis akajei (アカエイ), Rhinobatus Schlegeli (サカタザメ), Heterodontus japonicus (ネコザメ) beträchtlich stärker ist als bei den Knochenfischen außer Sparus swinhonis (クロダイ) und Pagrosomus major(マダイ). (Weil die Darstellung des ,Stiches ins Rote“ in der blauen Färbung in der weiß-schwarzen Photo unmöglich ist, wird hier die Abbildung nicht gebracht.) Bei den letzteren zwei Knochnfischen ist die Media an den mit Toluidinblau bei $\mathrm{pH} 4.1$ metachromatisch färbbaren sauren Polysaccharidverbindungen reich, deren ganze Menge fast die gleiche ist wie bei den Knorpelfischen.

Bei pH 2.5 ist aber keine Metachromasie bei beiden Fischarten zu sehen.

\section{Auswertung.}

TOKUDA (1957) hat zum ersten Mal auf die Mengenverhältnisse der Lipoide und Polysaccharidverbindungen im Blutplasma und Knorpelgrundsubstanz der Knochen- und Knorpelfische aufmerksam gemacht. Die vorliegende Untersuchung zeigt, daß auch ihre Menge in der Aortenwand bei beiden Fischarten verschieden ist. Die Lipoidmenge in ihr ist nämlich bei den Knochenfischen reichlicher, dagegen die der sauren Polysaccharidverbindungen bei den Knorpelfischen. Näher gesagt, finden sich die Lipoide bei den Knochenfischen in den elastischen Fasern, den Muskelfasern und der amorphen Grundsubstanz in der Media sowie in den elastischen Fasern der Adventitia reichlich, dagegen bei ihnen in der amorphen Grundsubstanz zwischen den faserigen und lamellenförmigen Elementen in der Adventitia geringer. Demgegenüber finden sie sich bei den Knorpelfischen sowohl in der Media als auch in der Adventitia sehr gering, nur daß die elastischen Fasern in der Adventitia mit den lipoidfärbenden Farbstoffen ziemlich stark angefärbt 
erscheinen.

Die Stärke der Pjs-SCHIFF-Reaktion und der Metachromasie mit Toluidinblau in der Arterienwand gehen merkwürdigerweise bei den untersuchten Fischarten nicht immer parallel einher. Diese Tatsache beruht offenbar darauf, daß die PjsSCHIFF-Reaktion nicth nur auf die sauren Polysaccharidverbindungen sondern auch auf Glykogen, neutrale Mukopolysaccharide, Glykoproteine, Cerebroside, Phosphatide usw. eintreten kann. Die Menge der bei pH 4.1 mit Toluidinblau metachromatisch färbbaren, als saure Polysaccharidverbindungen aufgefaßten Substanzen ist indes in der an Lipoiden reichen Aortenwand immer als reichlich zu beweisen, ausgenommen bei Sparus swinhonis (クロダイ) und Pagrosomus major (マダイ), bei welchen die Substanzen fast ebensoviel wie bei den Knorpelfischen nachgewiesen werden. Die Metachromasie mit Toluidinblau bei $\mathrm{pH} 4.1$ tritt bei Knochenfischen mäßig stark in der Media und etwas schwächer in der amorphen Grundsubstanz der Adventitia ein, während sie bei den Knorpelfischen schwach in der Media und auffallend stark in der amorphen Grnndsubstanz zwischen den Fasern in der Adventitia ansfällt.

Die bei pH 2.5 metachromatisch färbbare Chondroitinschwefelsäure wurde, wie in der Untersuchung von HAYASHI u. a. (1955) bei Mensch, Kaninchen und Ochsen, kaum in der Aortenwand der beiden Fischarten nachgewiesen.

\section{Zusammenfassung.}

1. Es wurde die Menge der mit mittelstark polaren Farbstoffen färbbaren lipoiden Substanzen in der Wand der Aorta ventralis von 14 Knochen- und 3 Knorpelfischarten vergleichend untersucht. Dieselbe wurde immer bei den Knochenfischen in reichlicherer Menge vorgefunden als bei den Knorpelfischen. Dagegen wurde die mit Toluidinblau bei pH 4.1 metachromatisch färbbaren, als sauren Polysaccharidverbindungen aufgefaßten Substanzen immer bei den Knorpelfischen mehr nachgewiesen als bei den Knochenfischen.

2. Die Pjs-SHIFF-Reaktion der Aortenwand fiel bei den Knochenfischen stärker oder fast in der gleicher Stärke aus wie bei den Knorpelfischen. Diese Reaktion stimmte bezüglich der Stärke nicht mit der metachromatischen Färbung mit Toluidinblau überein.

3. Die bei $\mathrm{pH} 2.5$ mit Toluidinblau metachromatisch zu färbende Substanz wurde kaum in der Aortenwand bei der Fischarten gefunden.

\section{内 容 自 抄.}

14 種の硬骨魚と 3 種の軟骨魚の腹側大動脉壁に含まれる類脂質の量を比較する と, その量は常に軟骨魚よりも硬骨魚に多い. しかるに $\mathrm{pH} 4.1$ にてトルイヂン 青で異調染する酸性多糖類化合物の量は常に軟骨魚に多い. 大動脉壁の過ヨード 酸 Schiff 反応は軟骨魚よりも硬骨魚に強いか, 又は殆ど等しく, pH 4.1 での異調 染色の強さと一致しない. pH 2.5 にては, 涵種の魚の大動脉壁ともトルイヂン青 で殆ど異調染色しない. 


\section{Literatur.}

Hayashi, H., K. Udaka, T. Funaki a. Y.Kato: Studies on acid mucopolysaccharides of the ground substance of connective tissue. I. Mie med. J. 4 (1955). Suppl. 2. - Kinukawa,K.: Beobachtung der Gewebe bei den täglich vorgenommene und langfristig durchgeführte intravenöse Injizierung von Natriumsulfidlösung erhaltenden Kaninchen. Arch. hist. jap. 12 (1957). - Kitade, T.: Über die Altersveränderungen der Aorta ventralis von Teleostier Goldfisch und über die Verschiedenheiten der Struktur der Aorta dorsalis von Selachier und Teleostier. Arch. hist. jap. 13 (1957). - Krause, R.: Mikroskopische Anatomie der Wirbeltiere. Bd. 4. 1923. - Kurita, H. : Über den Einfluß des Chondroitinsulfates auf die experimentelle Atherosklerose beim Kaninchen. Med. J. Shinshu Univ. 1 (1955). - Tokuda, M.: Über die Menge der Lipoide im Blutplasma von Knochen- und Knorpelfisch und die Stärke der Pjs-SCHIFF-Reaktion des Knorpels und seiner Metachromasie mit Toluidinblau. Arch. hist. jap. 12 (1957). - Die Korrelation zwischen der Höhe des Blutdruckes und der Konzentration des nach der Viktoriablaufiltrierpapiermethode bestimmbaren Lipoides im Blutplasma. Arch. hist. jap. 13 (1957). 


\section{Erläuterung zu den Tafelabbildungen.}

Querschnitte 1-16 sind aus der Aorta ventralis von Knochenfischen (links) und Knorpelfischen (rechtes). Ein paar Schnitte wurden auf ein und demselben Objektglas aufgeklebt und gefärbt.

Die Aortenschnitte in Abb. 1-6 sind mit lipoidfärbendem saurem Irisolechtviolett BBN gefärbt. $150 \times$

Abb. 1. Knochenfisch Scomber japonicus (ホンサバ) und Knorpelfisch Dasyatis akajei (アカエイ).

Abb. 2. Knochenfisch Trachurus japonicus (マアヂ) und Knorpelfisch Dasyatis akajei (アカエイ).

Abb. 3. Knochenfisch Sparus swinhonis (クロタイ) und Knorpelfisch Heterodontus japonicus (ネコザメ).

Abb. 4. Knochenfisch Mugil cephalus (マボラ) und Knorpelfisch Heterodontus japonicus (ネコザメ).

Abb. 5. Knochenfisch Cololabis saira (サンマ) und Knorpelfisch Rhinobatus S'chlegeli (サカタザメ).

Abb. 6. Knochenfisch Lateolabrax japonicus (スズキ) und Knorpelfisch Hetero. dontus japonicus (ネコザメ). 
R. HIROSE (1)
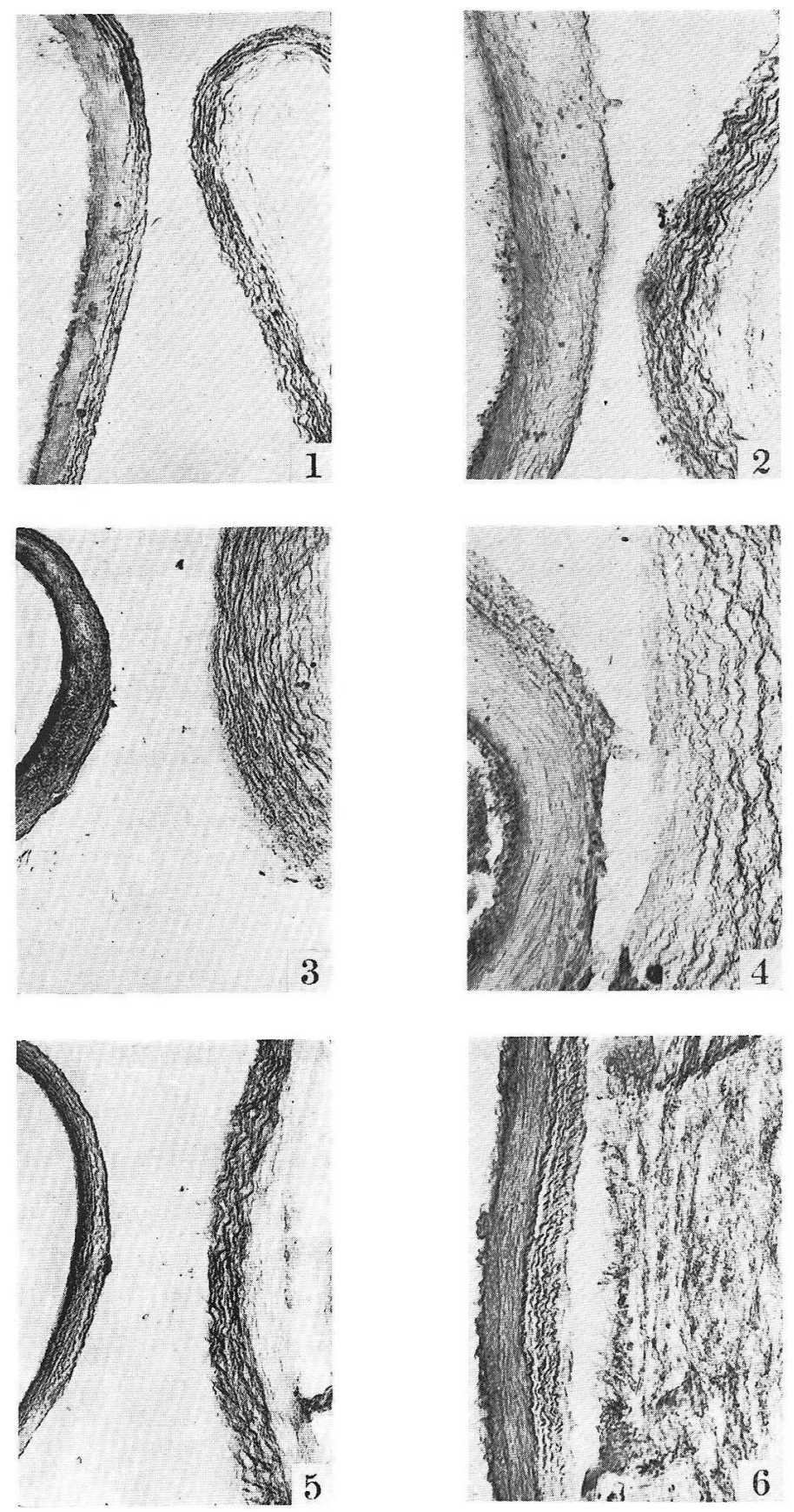
Die Aortenschnitte in Abb. 7--10 sind mit lipoidfärbendem basischem Viktoriablau gefärbt. $150 \times$

Abb. 7. Knochenfisch Scomber japonicus (ホンサバ) und Knorpelfisch Dasyatis akajei (アカエイ).

Abb. 8. Knochenfisch Trachurus japonicus (マアチ) und Knorpelfisch Dasyatis akajei (アカエイ).

Abb. 9. Knochenfisch Sparus swinhonis (クロタイ) und Knorpelfisch Heterodontus japonicus (ホコザメ).

Abb. 10. Knochenfisch Anguilla japonica (ウナギ) und Knorpelfisch Rhinobatus Schlegeli (サカタザメ). 
R. HIROSE (2)
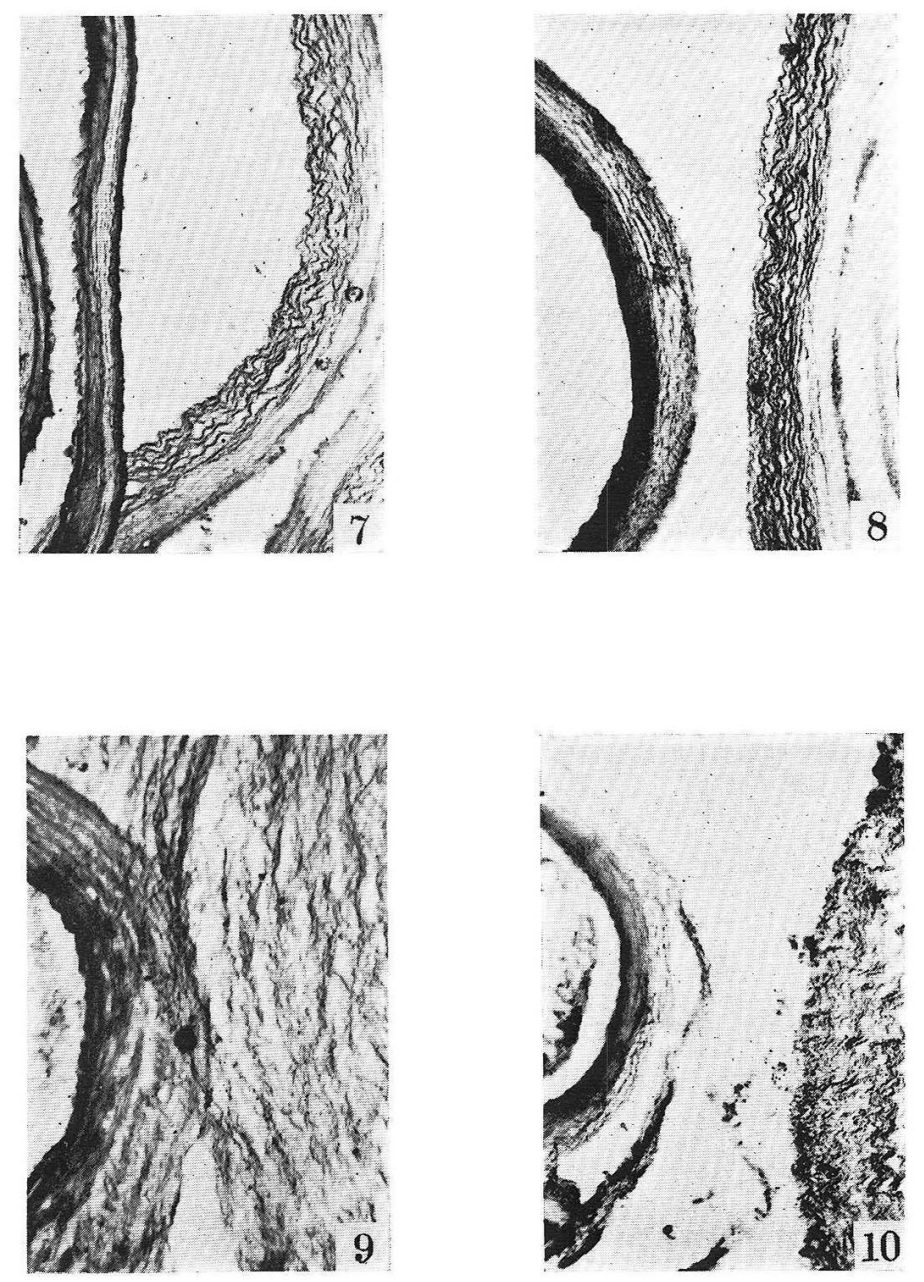
Die Abb. 11-16 geben der Pjs-SCHIFF-Reaktion unterworfene Aortenschnitte wieder. $150 \times$

Abb. 11. Knochenfisch Scomber japonicus (ホンサバ) und Knorpelfisch Dasyatis akajei (アカエイ).

Abb. 12. Knochenfisch Trachurus japonicus (マアヂ) und Knorpelfisch Dasyatis akajei (アカエイ).

Abb. 13. Knochenfisch Sparus swinhonis (クロタイイ) und Knorpelfisch Dasyatis akajei (アカエイ).

Abb. 14. Knochenfisch Cololabis saira (サンマ) und Knorpelfisch Dasyatis akajei (アカエイ).

Abb. 15. Knochenfisch Platycephalus indicus (コチ) und Knorpelfisch Rhinobatus Schlegeli (サカタザメ).

Abb. 16. Knochenfisch Pagrosomus major (マダイ) und Knorpelfisch Rhinobatus Schlegeli (サカタザメ). 
R. HIROSE ( 3)
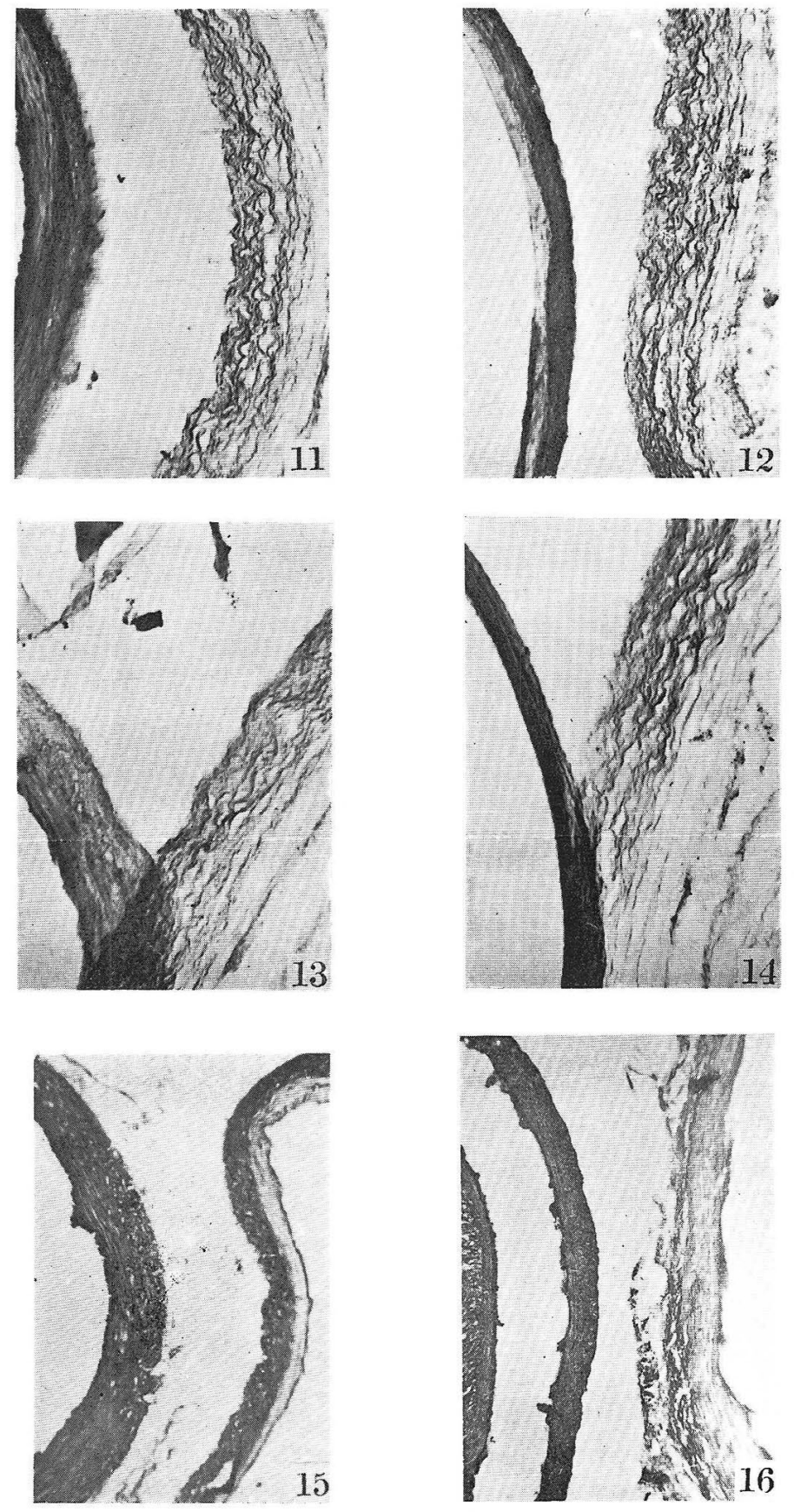